\title{
Diagnostic utility of plasma IncRNA small nucleolar RNA host gene 1 in patients with hepatocellular carcinoma
}

\author{
SHOUBAO GAO ${ }^{1}$, XIAOHONG XU ${ }^{2}$, YUE WANG $^{3}$, WEI ZHANG ${ }^{1}$ and XINGYE WANG ${ }^{2}$ \\ Departments of ${ }^{1}$ Oncology and ${ }^{2}$ Clinical Laboratory, The Third Affiliated Hospital of Qiqihar Medical University, \\ Qiqihar, Heilongjiang 161021; ${ }^{3}$ Medical School of Nanchang University, Nanchang, Jiangxi 330031, P.R. China
}

Received December 12, 2017; Accepted June 12, 2018

DOI: $10.3892 / \mathrm{mmr} .2018 .9336$

\begin{abstract}
Long non-coding RNAs (lncRNAs) offer great potential as cancer biomarkers. Owing to the limited sensitivity and specificity of $\alpha$-fetoprotein (AFP) for the diagnosis of hepatocellular carcinoma (HCC), the present study used an IncRNA microarray to screen aberrantly expressed lncRNAs in HCC tissues. Subsequently, the expression profile of the target IncRNAs was investigated in plasma from patients with HCC or hepatitis B virus-positive chronic hepatitis and cirrhosis $(\mathrm{HCH})$, as well as from healthy volunteers. A total of six aberrantly expressed lncRNAs were identified in HCC tissues and corresponding normal tissues, from which only small nucleolar RNA host gene 1 (SNHG1) expression in HCC tissues demonstrated a good correlation with those in plasma from HCC patients. Subsequent analysis revealed that high plasma SNHG1 expression levels were correlated with tumor size, TNM stage and AFP levels. Receiver operating characteristic analysis demonstrated that SNHG1 yields an excellent diagnostic ability to differentiate between patients with HCC and unaffected control patients, which was superior to that of AFP. The combination of SNHG1 with AFP may be able to distinguish $\mathrm{HCC}$ from $\mathrm{HCH}$ or healthy volunteers with the area under the curve values of 0.86 and 0.97 , respectively. In summary, it was demonstrated that plasma SNHG1 has great potential as a sensitive and reliable biomarker for the diagnosis of HCC.
\end{abstract}

Correspondence to: $\mathrm{Dr}$ Xiaohong Xu, Department of Clinical Laboratory, The Third Affiliated Hospital of Qiqihar Medical University, 27 Taishun Street, Qiqihar, Heilongjiang 161021, P.R. China E-mail: xuxiaohh067@163.com

Abbreviations: HCC, hepatocellular carcinoma; lncRNA, long non-coding RNA; SNHG1, small nucleolar RNA host gene 1; AFP, $\alpha$-fetoprotein; HBV, hepatitis B virus; $\mathrm{HCH}, \mathrm{HBV}$-positive chronic hepatitis and cirrhosis; ROC, receiver operating characteristic

Key words: hepatocellular carcinoma, long non-coding RNAs, small nucleolar RNA host gene 1, diagnosis, receiver operating characteristic analysis

\section{Introduction}

Hepatocellular carcinoma (HCC) is the fifth most common cause of cancer-associated mortality worldwide (1). It is a disease with a dismal outcome, and the 5-year overall survival rate is $<5 \%$ (2). Patients at the highest risk for developing HCC are those with chronic liver disease. The geographical variation in the incidence of HCC is explained by disparity in the prevalence of the major risk factors, such as hepatitis B virus (HBV) infection. For instance, the morbidity of HCC is high in Eastern Asia and sub-Saharan Africa, where HBV infection is endemic (3). There is a range of diagnostic criteria for $\mathrm{HCC}$, including index tumor detection, staging of intrahepatic tumor and assessment of extrahepatic metastasis (4). However, there is still a lack of convenient and reliable tumor markers in the diagnosis of HCC. $\alpha$-fetoprotein (AFP) expression is the most widely used tumor marker worldwide (5). An AFP level $>200 \mathrm{ng} / \mathrm{ml}$ in patients with cirrhosis has a very high positive predictive value for HCC (6). Conversely, up to $40 \%$ of HCC tissues never produce AFP, meaning that low AFP levels do not exclude HCC (7). The lack of knowledge regarding the molecular mechanisms of the development of HCC lead to delayed diagnosis and a high probability of relapse following treatment $(8,9)$. Therefore, the identification of reliable diagnostic markers for HCC is urgently required.

Long non-coding RNAs (lncRNAs) are molecules $>200$ bp long that do not encode protein products (10). Most of the currently known lncRNAs exert their function by participating in the regulation of a broad range of cellular processes at the epigenetic, transcriptional and post-transcriptional levels (11). A previous study has revealed that lncRNAs are frequently and aberrantly expressed in various cancers, and may serve potential roles as oncogenes and tumor suppressors (12). Notably, studies have also reported that deregulation of lncRNAs has potential significance for cancer diagnosis. Upregulated lncRNA cancer susceptibility candidate 15 is associated with poor prognosis in patients with HCC (13). Ubiquitin specific peptidase 16 is downregulated in $\mathrm{HCC}$ and functions as a tumor suppressor in HCC pathogenesis (14). In addition, serum UCA1 expression was identified as a noninvasive biomarker for HCC screening and prognostic prediction (15). Although these studies reported the crucial role of IncRNAs in the formation, progression and prognosis of $\mathrm{HCC}$, their expression profile and clinical significance in HCC remain largely unknown (16). 
Over the past few decades, microarray technology has been widely used to screen genetic alterations at the genome level (17). By performing lncRNA microarray analysis, Tang et al (18) demonstrated that lncRNAs RP11-160H22.5, XLOC_014172 and LOC149086 are upregulated in HCC, and they may be used as potential predictive biomarkers for tumorigenesis. Similarly, Cui et al (19) performed microarray analysis and identified lncRNAs PVT1 and SNHG7, which may be involved in HCC metastasis. The present study also used a lncRNA microarray assay to determine the differentially expressed lncRNAs between HCC tissues and corresponding adjacent normal tissues. The relationship between aberrant IncRNA expression between tissues and plasma was also analyzed. Expression levels of the lncRNA small nucleolar RNA host gene 1 (SNHG1) in HCC tissues exhibited a good correlation with those in plasma. Emerging evidence revealed that ectopic expression of SNHG1 functions as an oncogene in various cancers, including breast and lung cancer $(20,21)$. A recent study also identified that SNHG1 was upregulated in HCC cells, and promoted HCC cells proliferation and cycle progression (22). Moreover, although higher SNHG1 expression in HCC tissues indicated a poorer prognosis (22), circulating SNHG1 levels in plasma in patients with HCC and its diagnostic properties remain unclear. The present study aimed to investigate whether plasma SNHG1 may serve as a biomarker for $\mathrm{HCC}$ using receiver operating characteristic (ROC) curves and to further compare its diagnostic value with AFP.

\section{Materials and methods}

Subjects. A total of 172 individuals were enrolled in the present study, including 50 age- and sex-matched healthy subjects (age range, 45-69; Control group), 50 patients with HBV-positive chronic hepatitis and cirrhosis (age range, 39-73; HCH group) and 72 patients with $\mathrm{HCC}$ (age range, 42-71; HCC group) from the Third Affiliated Hospital of Qiqihar Medical University (Qiqihar, China) between January 2015 and December 2016. Clinical data were collected, and the main demographic and clinical characteristics of the studied subjects are provided in Table I. Blood samples from all subjects prior to any medical interventions were collected into EDTA anti-coagulation tubes and processed for plasma extraction within $2 \mathrm{~h}$ of collection (centrifuged at $3,000 \mathrm{x}$ g for $10 \mathrm{~min}$ at $4^{\circ} \mathrm{C}$ ). Blood samples following surgery from patients with $\mathrm{HCC}$ were also collected. The plasma was stored at $-80^{\circ} \mathrm{C}$ in polypropylene tubes for further analysis. Tumor tissues and adjacent normal tissues from the 72 patients with HCC were collected during surgery at the Third Affiliated Hospital of Qiqihar Medical University. All procedures were conducted in accordance with the protocol that was approved by the Ethics Committee of the Third Affiliated Hospital of Qiqihar Medical University, and written informed consent was obtained from each subject. Patients who underwent previous preoperative chemotherapy or radiotherapy were excluded.

Biochemical analysis. The levels of alanine transaminase (ALT), aspartate aminotransferase (AST), albumin, total bilirubin and glucose were measured using an automated biochemistry analyzer (AU5800; Beckman Coulter, Inc., Brea,
CA, USA). The levels of plasma $\alpha$-fetoprotein (AFP) were detected with an enzyme-linked immunosorbent assay kit (cat. no. XF00419B; Shanghai Xinfan Biotechnology Co., Ltd., Shanghai, China) according to the manufacturer's instructions. Signals were determined by measuring the absorbance at $450 \mathrm{~nm}$ using a spectraMax M series multi-mode microplate reader (Molecular Devices, San Jose, CA, USA).

RNA isolation and microarray analysis. Total RNA from six random tumor tissues and adjacent normal tissues was extracted using TRIzol reagent (Invitrogen; Thermo Fisher Scientific, Inc., Waltham, MA, USA) according to the manufacturer's protocol (23). RNA quality was confirmed by formaldehyde agarose gel electrophoresis and quantified by NanoDrop ND-2000 (Thermo Fisher Scientific, Inc.). RNA was used to synthesize double-stranded cDNA using SuperScript Double-Stranded cDNA Synthesis kit (Thermo Fisher Scientific, Inc.), which was stored at $-80^{\circ} \mathrm{C}$ for further use. The RT reaction was performed at $95^{\circ} \mathrm{C} 15 \mathrm{sec}$, followed by 30 cycles of $95^{\circ} \mathrm{C}$ for $5 \mathrm{sec}$ and $60^{\circ} \mathrm{C}$ for $1 \mathrm{~min}$. cDNA was labeled with $\mathrm{Cy} 3$ or $\mathrm{Cy} 5$ fluorescent probes (Aksomics, Inc., Shanghai, China) and hybridized to the Human LncRNA Expression Microarray v3.0 (format, 8x60 K; Arraystar, Rockville,MD,USA), according to the manufacturer's protocol. Following washing with a NimbleGen Wash kit (Roche Diagnostics, Basel, Switzerland), the slides were scanned with an Agilent Microarray Scanner (Agilent Technologies, Inc., Santa Clara, CA, USA), and data were analyzed with ImaGene software (version 9.0; BioDiscovery, Inc., El Segundo, CA, USA) on the scanner and extracted as paired files. Significant differences were calculated using the paired Student's t-test. Hierarchical clustering was conducted to demonstrate the aberrantly expressed lncRNAs via Cluster-TreeView software (version 3.0; Palo Alto, CA, USA) (24).

Reverse transcription-quantitative polymerase chain reaction (RT-qPCR). Total RNA was extracted using TRIzol reagent (Invitrogen; Thermo Fisher Scientific, Inc.). First strand cDNA was synthesized from 72 tissue or plasma samples (50 samples in the control group and $\mathrm{HCH}$ group, 72 samples in the HCC group) using a PrimeScript RT Reagent Kit (Takara Biotechnology Co., Ltd., Dalian, China), according to the manufacturer's protocol; the reaction was performed at $16^{\circ} \mathrm{C}$ for $30 \mathrm{~min}, 42^{\circ} \mathrm{C}$ for $30 \mathrm{~min}$ and $85^{\circ} \mathrm{C}$ for $5 \mathrm{~min}$. qPCR was performed to quantify the expression level of lncRNA with SYBR Premix Ex Taq (Takara Biotechnology Co., Ltd.) and the ABI Prism 7900HT Fast Real-Time PCR System (Applied Biosystems; Thermo Fisher Scientific, Inc.). PCR was performed at $95^{\circ} \mathrm{C}$ for $2 \mathrm{~min}$, followed by 40 cycles at $94^{\circ} \mathrm{C}(15 \mathrm{sec}), 60^{\circ} \mathrm{C}(60 \mathrm{sec})$ and $72^{\circ} \mathrm{C}(30 \mathrm{sec})$. All experiments were carried out in triplicate. Relative expression levels were calculated using the $2^{-\Delta \Delta \mathrm{Cq}}$ method (25) and were normalized to GAPDH. The primers used are provided in Table II.

Statistical analysis. Statistical analysis was performed using SPSS 19.0 software (IBM Corp., Armonk, NY, USA) and GraphPad Prism 5.5 (GraphPad Software, Inc., La Jolla, CA, USA). Continuous variables were expressed as the mean \pm standard deviation, and categorical variables were presented as frequencies. All enrolled subjects were assigned 
Table I. Clinicopathological characteristics of subjects in the present study.

\begin{tabular}{lccrr}
\hline Clinicopathological features & HCC $(\mathrm{n}=72)$ & $\mathrm{HCH}(\mathrm{n}=50)$ & Control (n=50) & P-value \\
\hline Age (years) & $51.26 \pm 7.31$ & $49.23 \pm 8.06$ & $50.37 \pm 7.19$ & 0.341 \\
Sex (male/female) & $57 / 15$ & $39 / 11$ & $35 / 15$ & 0.473 \\
Smoking & 46 & 33 & 27 & 0.408 \\
Alcoholism & 26 & 18 & $24.52 \pm 10.94$ & 0.701 \\
ALT (U/l) & $70.12 \pm 45.72$ & $79.56 \pm 63.46$ & $21.33 \pm 8.47$ & $<0.001$ \\
AST (U/l) & $71.06 \pm 50.38$ & $63.22 \pm 47.19$ & $39.72 \pm 5.14$ & $<0.001$ \\
Albumin (g/dl) & $37.80 \pm 4.66$ & $38.25 \pm 6.71$ & $15.24 \pm 4.60$ & 0.154 \\
Total bilirubin (mg/dl) & $23.73 \pm 10.16$ & $29.57 \pm 48.36$ & $4.87 \pm 1.16$ & 0.031 \\
Glucose (mmol/l) & $5.04 \pm 1.21$ & $4.98 \pm 1.09$ & $21.51 \pm 6.42$ & 0.729 \\
AFP & $2,036.72 \pm 418.57$ & $44.57 \pm 89.33$ & $<0.001$
\end{tabular}

AFP, $\alpha$-fetoprotein; ALT, alanine transaminase; AST, aspartate aminotransferase; HBV, hepatitis B virus; HCC, hepatocellular carcinoma; $\mathrm{HCH}, \mathrm{HBV}$-positive chronic hepatitis and cirrhosis.

Table II. Forward and reverse primer sequences for reverse transcription-quantitative polymerase chain reaction.

\begin{tabular}{ll}
\hline Gene & \multicolumn{1}{c}{ Primer sequence $\left(5^{\prime} \rightarrow 3 '\right)$} \\
\hline BF896662 & F: TGCACCAGTTCAGAGCCAGAG \\
& R: ACAGATGATGGTATGATGAC \\
GSO_1539211_385 & F: GATGTTCTTGCTGTGGTGGTT \\
& R: ACAGTCTCATCGGCTGATTG \\
ASLNC12707 & F: CTCGCGAGAGACGACATTCG \\
& R: GGTAGGGAATCGAGGAGAATG \\
XLOC_002237 & F: AGCCACTGGAGAAGTGTCACC \\
& R: CATTCGTTGGCCACGTCCATT \\
XLOC_014001 & F: CTCGGCCAGCATGTCGT \\
& R: ATCGCTACACGATGCATACT \\
ASLNC12773 & F: CTTAGAACACGGTCTAACGA \\
& CTT \\
& R: GTTGCAACTCCTGGTCACCTGC \\
FNHG1 & R: CAGCCTGCTTGGCTCAAAGGG \\
F: CTCAGACTGAGAGACATATCCA & FGA
\end{tabular}

DGCR5, DiGeorge syndrome critical region gene 5; SNHG1, small nucleolar RNA host gene 1.

to high and low SNHG1 groups, according to the mean value of plasma SNHG1. The differences between SNHG1 expression and clinical characteristics were analyzed by $\chi^{2}$, and a paired Student's t-test was used to compare the differences between plasma SNHG1 expression levels pre- and post-surgery. The differences in SNHG1, ASLNC12773 and BF896662 expression levels among the $\mathrm{HCC}, \mathrm{HCH}$ and Control groups was analyzed by a one-way analysis of variance, followed by the least significance difference test. Correlation between plasma and tissue SNHG1 levels was analyzed by Pearson correlation analysis. ROC curves were constructed, and the area under the ROC curve (AUC) was generated to assess the diagnostic performance of SNHG1. $\mathrm{P}<0.05$ was considered to indicate a statistically significant difference.

\section{Results}

SNHG1 expression is higher in tumoral tissues and plasma of patients with HCC. To determine the lncRNAs profiling in HCC tissues, lncRNA microarray analysis of six paired HCC and adjacent normal tissues was performed. Hierarchical clustering demonstrated systematic variations in transcript expression levels between the six paired tissues (Fig. 1A). A total of 357 lncRNAs were identified to be differentially expressed, and the top 5 upregulated and the top 5 downregulated lncRNAs in HCC are listed in Table III. These aberrantly expressed lncRNAs were further validated in all $72 \mathrm{HCC}$ and adjacent normal tissues using RT-qPCR, and the results demonstrated that four of them demonstrated no significant difference between the tissues (Fig. 1B). Additionally, the expression levels of six identified lncRNAs were subsequently investigated. Among these, ASLNC12707,GSO_1539211_385 and XLOC_014001 were not detectable in the plasma and were excluded from further study. The relative expression levels of SNHG1 and ASLNC12773 were significantly higher in patients with $\mathrm{HCH}$ compared with the respective expression levels in healthy Control patients (Fig. 2A and B, respectively), but only SNHG1 was expressed at significantly higher levels in $\mathrm{HCC}$ compared with $\mathrm{HCH}$ tissue (Fig. 2A). Conversely, the expression level of BF896662 was lower in patients with $\mathrm{HCH}$ compared with healthy Control individuals (Fig. 2C); however, no statistically significant difference was identified between patients with $\mathrm{HCC}$ and $\mathrm{HCH}$. A 
A
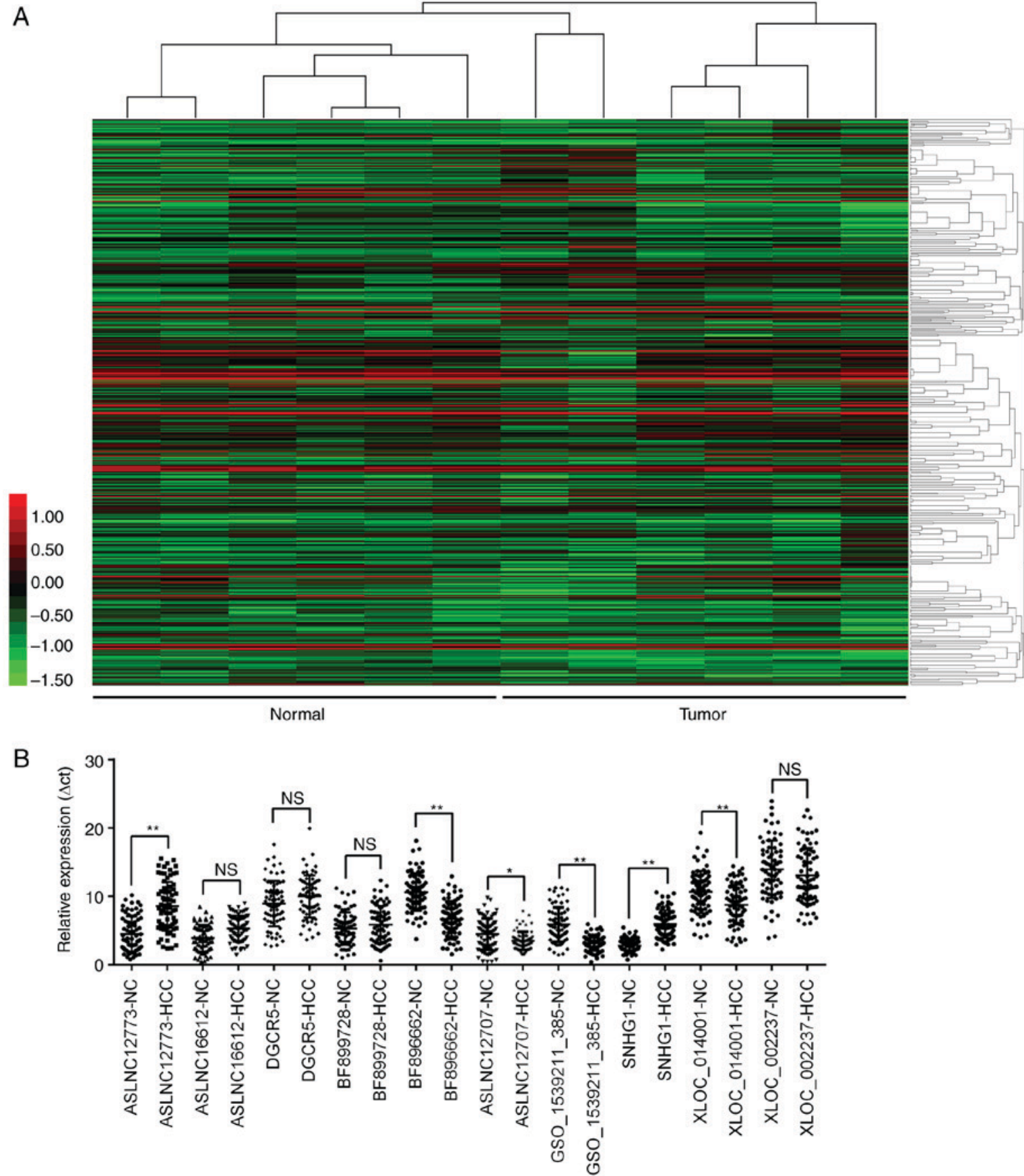

Figure 1. IncRNA expressions in HCC tissues. (A) lncRNA expression spectrum clustering map from six paired tumor samples and adjacent normal tissues. Green and red bars indicate downregulation and upregulation, respectively. (B) Relative expression levels of 10 aberrantly expressed lncRNAs were validated by reverse transcription-quantitative polymerase chain reaction. ${ }^{*} \mathrm{P}<0.05$ and ${ }^{* *} \mathrm{P}<0.01$. NC, negative control from the corresponding adjacent normal tissue. DGCR5, DiGeorge syndrome critical region gene 5, HCC, hepatocellular carcinoma; lncRNA, long non-coding RNA; NS, not significant; SNHG1, small nucleolar RNA host gene 1.

positive correlation was demonstrated between plasma and HCC tissue SNHG1 expression levels ( $r=0.66$; Fig. 2D). Owing to its stability in plasma and its ability to differentiate HCC and $\mathrm{HCH}$, the plasma levels of SNHG1 were compared pre- and post-surgery (Fig. 2E). Plasma SNHG1 expression was notably reduced following surgery compared with expression levels prior to surgery (Fig. 2E), which suggested that SNHG1 may have been released from HCC tissues into the bloodstream.

Association between SNHG1 expression and clinicopathological characteristics. The relationship between plasma SNHG1 expression level and the clinicopathological characteristics of the present cohort was analyzed (Table IV). High SNHG1 expression was significantly associated with tumor size $(\mathrm{P}=0.047)$, TNM stage $(\mathrm{P}=0.012)$ and AFP level $(\mathrm{P}=0.024)$. By contrast, SNHG1 expression did not demonstrate an association with other clinical factors, including age, sex, smoking status, cirrhosis and tumor number (all $\mathrm{P}>0.05$ ).

Diagnostic performance of SNHG1 in patients with HCC. ROC curves were constructed and the AUC was generated to assess the diagnostic performance of SNHG1 in HCC. ROC 
Table III. Top 5 upregulated and top 5 downregulated lncRNAs in hepatocellular carcinoma tissues identified by microarray analysis.

\begin{tabular}{lrrr}
\hline Gene ID & $\log 2(\mathrm{FC})$ & P-value & Regulation \\
\hline BF896662 & -2.433374357 & 0.00053002 & Down \\
GSO_1539211_385 & -2.167898147 & 0.00015589 & Down \\
ASLNC12707 & -1.975377648 & 0.00008775 & Down \\
XLOC_002237 & -1.830784055 & 0.00020238 & Down \\
XLOC_014001 & -1.707527413 & 0.00010118 & Down \\
ASLNC12773 & 3.298459185 & 0.00050628 & Up \\
SNHG1 & 2.845947285 & 0.00023835 & Up \\
BF899728 & 2.541892168 & 0.00004255 & Up \\
ASLNC16612 & 2.503652367 & 0.00040132 & Up \\
DGCR5 & 2.348980639 & 0.00185469 & Up
\end{tabular}

DGCR5, DiGeorge syndrome critical region gene 5; FC, fold change; lncRNA, long non-coding RNA; SNHG1, small nucleolar RNA host gene 1 .
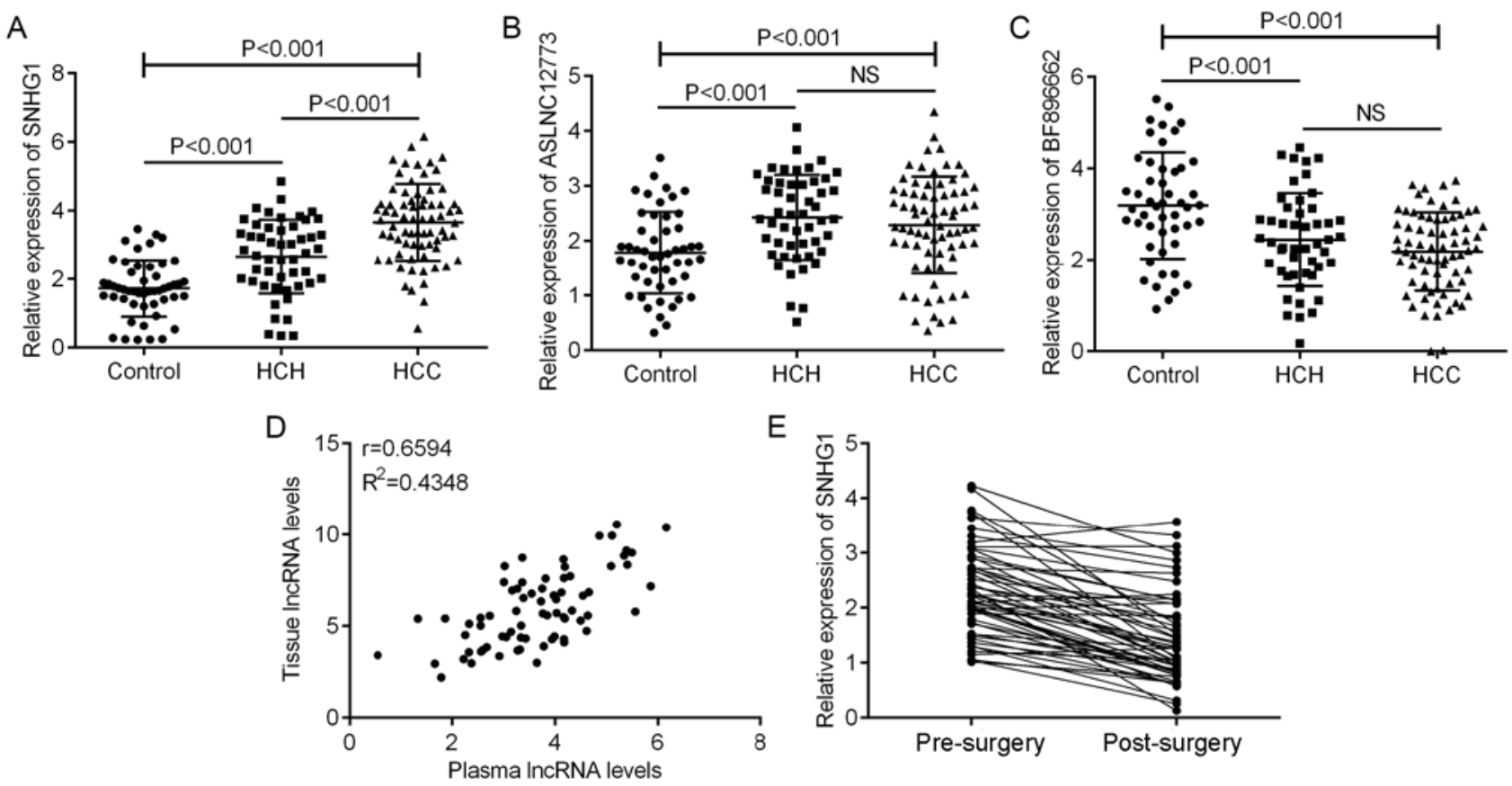

Figure 2. Relative expression levels of 1ncRNA SNHG1 in plasma samples from patients with HCC, patients with HCH and healthy Control individuals. (A-C) Relative lncRNA expression levels were determined by reverse transcription-quantitative polymerase chain reaction for (A) SNHG1, (B) ASLNC12773 and (C) BF896662 in the three groups. (D) A positive correlation was identified between plasma and tissue SNHG1 expression by Pearson correlation analysis. $r=0.6210 ; R^{2}=0.3857 ; P<0.001$. (E) Relative plasma expression levels of SNHG1 pre- and post-surgery in 72 patients with HCC. HCC, hepatocellular carcinoma; $\mathrm{HCH}$, hepatitis B virus-positive chronic hepatitis and cirrhosis; lncRNA, long non-coding RNA; NS, not significant; SNHG1, small nucleolar RNA host gene 1.

analyses demonstrated that the AUC values of plasma SNHG1 and AFP were 0.92 [95\% confidence interval (CI), 0.86-0.96] and 0.85 (95\% CI, 0.77-0.90), respectively, to distinguish HCC from controls (Table V; Fig. 3A). The cut-off values of SNHG1 and AFP were 2.54 and $187.88 \mu \mathrm{g} / 1$, respectively. When distinguishing between $\mathrm{HCC}$ and $\mathrm{HCH}$, the AUC values of SNHG1 and AFP were 0.74 (95\% CI, 0.65-0.83) and 0.79 (95\% CI, 0.71-0.86), respectively (Table V; Fig. 3B). Accordingly, the cut-off values of SNHG1 and AFP were 3.25 and $268.11 \mu \mathrm{g} / \mathrm{l}$, respectively. In addition, the combination of SNHG1 and AFP possessed higher AUC values for the discrimination between
HCC patients and controls as compared with SNHG1 alone, with higher sensitivity (Table V; Fig. 3C). The combination also possessed a higher AUC values for discrimination between HCC and $\mathrm{HCH}$ patients with higher specificity (Table V; Fig. 3D). These data indicated that the combination of SNHG1 and AFP achieved a better diagnostic accuracy than AFP alone.

\section{Discussion}

Despite great advances in the diagnosing HCC, many patients are still diagnosed with HCC at an advanced stage (26), as 
Table IV. Clinicopathological characteristics of the patients, divided into two groups according to the plasma lncRNA SNHG1 levels in HCC.

\begin{tabular}{|c|c|c|c|c|}
\hline \multirow[b]{2}{*}{ Clinicopathological characteristics } & \multirow[b]{2}{*}{ Total $(\mathrm{n}=72)$} & \multicolumn{2}{|c|}{ Plasma SNHG1 expression } & \multirow[b]{2}{*}{ P-value } \\
\hline & & Low $(n=39)$ & High $(n=33)$ & \\
\hline Age & & & & 0.605 \\
\hline$<50$ & 47 & 27 & 20 & \\
\hline$\geq 50$ & 25 & 12 & 13 & \\
\hline Sex & & & & 0.258 \\
\hline Male & 44 & 21 & 23 & \\
\hline Female & 28 & 18 & 10 & \\
\hline Smoker & & & & 0.401 \\
\hline Yes & 30 & 14 & 16 & \\
\hline No & 42 & 25 & 17 & \\
\hline Cirrhosis & & & & 0.109 \\
\hline Yes & 33 & 14 & 19 & \\
\hline No & 39 & 25 & 14 & \\
\hline Tumor size $(\mathrm{cm})$ & & & & 0.047 \\
\hline$<5$ & 32 & 22 & 10 & \\
\hline$\geq 5$ & 40 & 17 & 23 & \\
\hline Tumor number & & & & 0.722 \\
\hline Single & 61 & 32 & 29 & \\
\hline Multiple & 11 & 7 & 4 & \\
\hline TNM stage & & & & 0.012 \\
\hline I-II & 49 & 32 & 17 & \\
\hline III-IV & 23 & 7 & 16 & \\
\hline $\operatorname{AFP}(\mu \mathrm{g} / \mathrm{l})$ & & & & 0.024 \\
\hline$<200$ & 46 & 30 & 16 & \\
\hline$\geq 200$ & 26 & 9 & 17 & \\
\hline
\end{tabular}

AFP, $\alpha$-fetoprotein; HCC, hepatocellular carcinoma; lncRNA, long non-coding RNA; SNHG1, small nucleolar RNA host gene 1; TNM, tumor, node, metastasis.

techniques, such as imaging and histology, only work at the late stages (27). To extend the time window for early diagnosis, additional investigations should be made into circulating biomarker testing. AFP has been extensively studied as a biomarker for diagnosis and prognosis in patients with HCC, but it has low accuracy and a high rate of false positives (28). A number of previous studies have reported that lncRNAs may be used as biomarkers for predicting survival and metastasis, or diagnosis, in a number of diseases, including HCC. For example, the lncRNA differentiation antagonizing non-protein coding RNA (DANCR) was determined to be higher in the plasma of patients with HCC compared with DANCR levels in healthy individuals and non-HCC patients, and subsequent diagnostic evaluation identified DANCR as a reliable biomarker for HCC diagnosis (29). Another study identified circulating lncRNA SPRY4-intronic transcript 1 as a good diagnostic value for HCC and its diagnostic property was increased when in combination with AFP (30). In addition, LINC00152, RP11-160H22.5 and XLOC014172 were reported as fingerprints for the early identification of HCC (31). The above findings provided the evidence that
lncRNAs may be a promising diagnostic target for early $\mathrm{HCC}$ diagnosis.

In the present study, six aberrantly expressed lncRNAs in paired HCC tissues and adjacent normal ones were investigated, through a microarray and RT-qPCR. We The present study focused on the top five up and downregulated lncRNAs identified in the collected HCC tissues. Their expression levels were investigated in the plasma of patients with HCC and two other groups of patients without HCC to evaluate their diagnostic values. Apart from those undetectable in the plasma and which demonstrated no significant differences among groups, only plasma SNHG1 expression was higher in HCC compared with $\mathrm{HCH}$ and healthy Control group plasma. High SNHG1 expression in HCC tissues has been reported in previous studies, and it was demonstrated to function as an oncogene in HCC (32), and the clinical prognostic significance of SNHG1 in HCC has been demonstrated (22). However, the expression profile of SNHG1 in plasma and its diagnostic value remained unclear. Consistent with previous findings, the present study revealed a significant increase of SNHG1 in HCC tissues, in comparison with its level in non-tumoral tissues, and plasma 
Table V. Receiver operating characteristic analysis of lncRNA in HCC patients.

\begin{tabular}{|c|c|c|c|c|}
\hline Group & AUC (95\%CI) & $\mathrm{P}$ value & Sensitivity (\%) & Specificity (\%) \\
\hline \multicolumn{5}{|l|}{ SHNG1 } \\
\hline HCC vs. Control & $0.92(0.86-0.96)$ & $<0.001$ & 87.3 & 86.0 \\
\hline HCC vs. HCH & $0.74(0.65-0.83)$ & $<0.001$ & 70.1 & 68.2 \\
\hline \multicolumn{5}{|l|}{ AFP } \\
\hline HCC vs. Control & $0.85(0.77-0.90)$ & $<0.001$ & 64.6 & 94.6 \\
\hline HCC vs. HCH & $0.79(0.71-0.86)$ & $<0.001$ & 71.6 & 74.0 \\
\hline \multicolumn{5}{|c|}{ Combined (SHNG1 + AFP) } \\
\hline HCC vs. Control & $0.97(0.92-0.99)$ & $<0.001$ & 96.4 & 87.0 \\
\hline HCC vs. HCH & $0.86(0.78-0.91)$ & $<0.001$ & 73.4 & 86.1 \\
\hline
\end{tabular}

AFP, $\alpha$-fetoprotein; HCC, hepatocellular carcinoma; $\mathrm{HCH}$, hepatitis B virus-positive chronic hepatitis and cirrhosis; lncRNA, long non-coding RNA; ROC, receiver operating characteristic; SNHG1, small nucleolar RNA host gene 1.
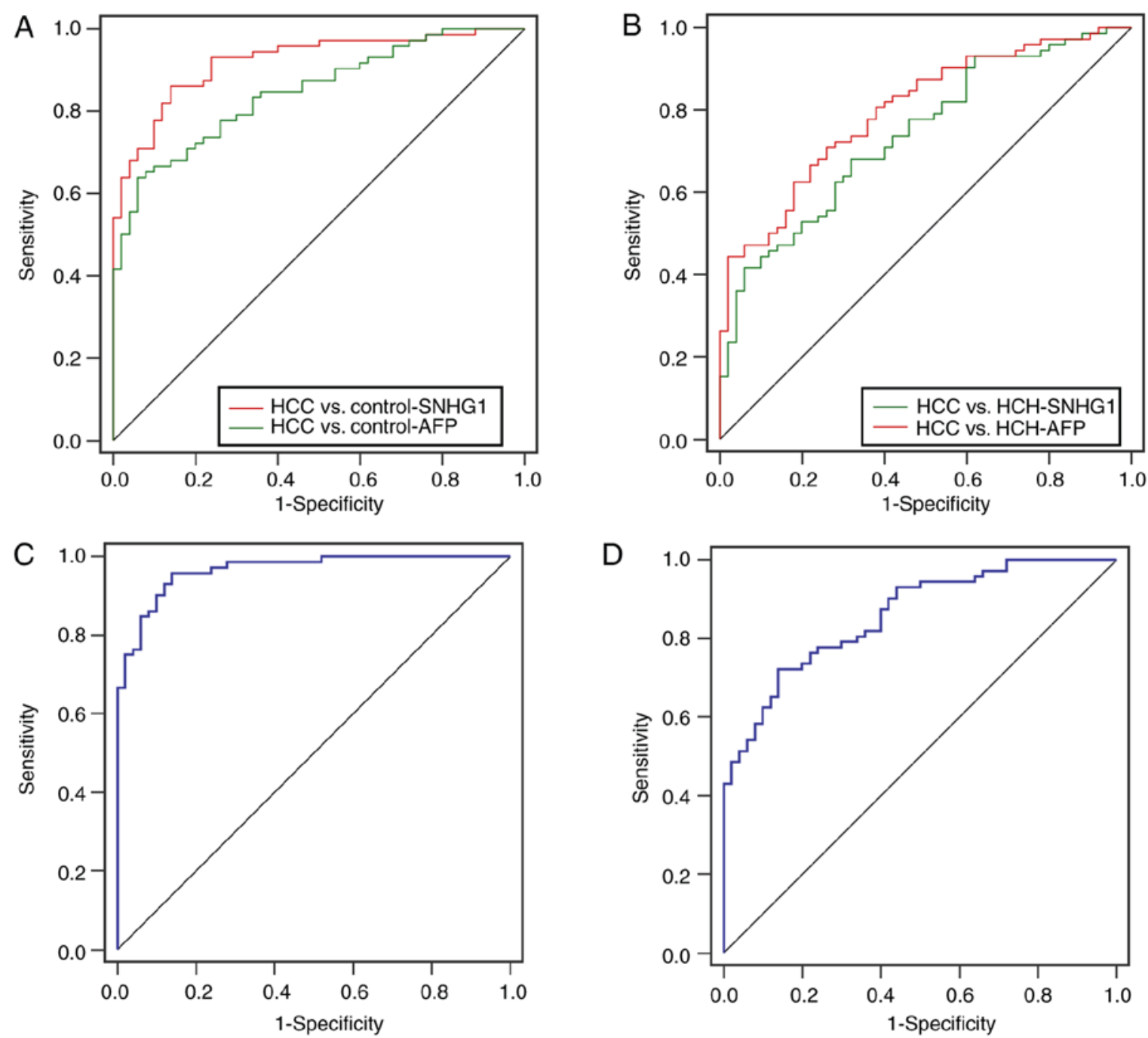

Figure 3. Diagnostic performance of plasma SNHG1 and AFP. (A) ROC curve analysis of plasma SNHG1 and AFP expression in differentiating patients with HCC $(n=72)$ from healthy controls $(n=50)$. SNHG1, AUC $=0.92(95 \%$ CI, 0.86-0.96), cut-off value $=2.54$; AFP, AUC $=0.85$ (95\% CI, 0.77-0.90), cut-off value $=187.88 \mu \mathrm{g} / 1$. (B) ROC analysis of plasma SNHG1 and AFP expression in differentiating patients with HCC ( $\mathrm{n}=72)$ from patients with HCH (n=50). SNHG1, AUC $=0.74$ (95\% CI, 0.65-0.83), cut-off value $=3.25$; AFP, AUC $=0.79(95 \%$ CI, 0.71-0.86), cut-off value $=268.11 \mu \mathrm{g} / \mathrm{l}$. (C) ROC analysis of combination of SNHG1 and AFP differentiate patients with HCC from health Controls. (D) ROC analysis of the combination of SNHG1 and AFP differentiate patients with HCC from patients with HCH. AFP, $\alpha$-fetoprotein; AUC, area under the ROC curve; CI, confidence interval; HCC, hepatocellular carcinoma; HCH, hepatitis B virus-positive chronic hepatitis and cirrhosis; lncRNA, long non-coding RNA; NS, not significant; ROC, receiver operating characteristic; SNHG1, small nucleolar RNA host gene 1. 
SNHG1 expression was positively correlated with SNHG1 expression in tissues; SNHG1 expression was demonstrated to be decreased following surgery, which suggested that circulating SNHG1 may have originated from HCC tissues. These results also demonstrated that SNHG1 may serve a role in monitoring recurrence following surgery.

Subsequently, the diagnostic value of SNHG1 was investigated using ROC analysis. The results indicated that SNHG1 may have an excellent diagnostic ability to differentiate between patients with HCC and healthy controls, which was better than AFP. However, when it comes to distinguishing $\mathrm{HCC}$ from $\mathrm{HCH}$, SNHG1 demonstrated a moderate diagnostic performance, similar to AFP. Certain patients with HCC may have developed the disease from HBV infection, and many may be suffering with chronic hepatitis or liver cirrhosis (33). Biomarkers for distinguishing $\mathrm{HCC}$ from $\mathrm{HCH}$ may be a useful clinical approach. Therefore, both features were used in the diagnostic procedure. As expected, the combination of SNHG1 with AFP achieved a better diagnostic accuracy.

However, the present study has limitations. Firstly, the sample size was small and these preliminary findings should be validated in trials with more subjects. Secondly, although SNHG1 was detectable and stable in plasma, the mechanisms underlying its secretion and transport to the circulation are poorly understood. Thirdly, the biological functions of SNHG1 in HCC were not investigated in vitro. However, it has been previously demonstrated that SNHG1 promotes HCC cell proliferation, cell cycle progression and inhibited apoptosis in vitro $(22,32)$. Finally, because the prognostic value of SNHG1 in tissues was reported by other studies (34-36), and the present study demonstrated that increased plasma SNHG1 was correlated with tumor size and TNM stage, it was hypothesized that plasma SNHG1 may serve as a biomarker for monitoring HCC following surgery.

In conclusion, the present study data are the first, to the best of our knowledge, to demonstrate that plasma SNHG1 was significantly higher in patients with HCC compared with expression levels in patients with $\mathrm{HCH}$ or healthy individuals. Increased plasma SNHG1 expression may be a valuable biomarker for HCC diagnosis. However, prospective studies with larger sample sizes are required to confirm these findings.

\section{Acknowledgements}

Not applicable.

\section{Funding}

The present study was supported by The Science and Technology Project of Qiqihar City (grant no. SFGG-201652).

\section{Availability of data and materials}

The analyzed data sets generated during the study are available from the corresponding author on reasonable request.

\section{Authors' contributions}

XX provided the funding and designed the study. YW and WZ collected the samples from the subjects and performed reverse transcription-quantitative polymerase chain analysis. SG and XW performed the microarray analysis. SG and YW performed the statistical analysis. SG and XX wrote the manuscript.

\section{Ethics approval and consent to participate}

The present study was approved by the Ethics Committee of the Third Affiliated Hospital of Qiqihar Medical University (Qiqihar, China), and written informed consent was obtained from each participant prior to enrolment in the study.

\section{Patient consent for publication}

Not applicable.

\section{Competing interests}

The authors declare that they have no competing interests.

\section{References}

1. Siegel RL, Miller KD and Jemal A: Cancer Statistics, 2017. CA Cancer J Clin 67: 7-30, 2017.

2. Dhanasekaran R, Limaye A and Cabrera R: Hepatocellular carcinoma: Current trends in worldwide epidemiology, risk factors, diagnosis, and therapeutics. Hepat Med 4: 19-37, 2012.

3. Wang CH, Wey KC, Mo LR, Chang KK, Lin RC and Kuo JJ: Current trends and recent advances in diagnosis, therapy, and prevention of hepatocellular carcinoma. Asian Pac J Cancer Prev 16: 3595-3604, 2015.

4. Arslanoglu A, Seyal AR, Sodagari F, Sahin A, Miller FH, Salem R and Yaghmai V: Current guidelines for the diagnosis and management of hepatocellular carcinoma: A comparative review. AJR Am J Roentgenol 207: W88-W98, 2016.

5. Wang Y,Jing W, Ma W, Liang C, Chai Hand Tu J: Down-regulation of long non-coding RNA GAS5-AS1 and its prognostic and diagnostic significance in hepatocellular carcinoma. Cancer Biomark 2018.

6. Tateishi R, Yoshida H, Matsuyama Y, Mine N, Kondo Y and Omata M: Diagnostic accuracy of tumor markers for hepatocellular carcinoma: A systematic review. Hepatol Int 2: 17-30, 2008.

7. Ferenci P, Fried M, Labrecque D, Bruix J, Sherman M, Omata M, Heathcote J, Piratsivuth T, Kew M, Otegbayo JA, et al: World gastroenterology organisation guideline. Hepatocellular carcinoma (HCC): A global perspective. J Gastrointestin Liver Dis 19: 311-317, 2010

8. Earl TM and Chapman WC: Conventional surgical treatment of hepatocellular carcinoma. Clin Liver Dis 15: 353-370, vii-x, 2011.

9. Li L, Lei Q, Zhang S, Kong L and Qin B: Screening and identification of key biomarkers in hepatocellular carcinoma: Evidence from bioinformatic analysis. Oncol Rep 38: 2607-2618, 2017.

10. Ponting CP, Oliver PL and Reik W: Evolution and functions of long noncoding RNAs. Cell 136: 629-641, 2009.

11. Mercer TR, Dinger ME and Mattick JS: Long non-coding RNAs: insights into functions. Nat Rev Genet 10: 155-159, 2009.

12. Isin M and Dalay N: LncRNAs and neoplasia. Clin Chim Acta 444: 280-288, 2015.

13. He T, Zhang L, Kong Y, Huang Y, Zhang Y, Zhou D, Zhou X, Yan Y, Zhang L, Lu S, et al: Long non-coding RNA CASC15 is upregulated in hepatocellular carcinoma and facilitates hepatocarcinogenesis. Int J Oncol 51: 1722-1730, 2017.

14. Zheng ZK, Pang C, Yang Y, Duan Q, Zhang J and Liu WC: Serum long noncoding RNA urothelial carcinoma-associated 1: A novel biomarker for diagnosis and prognosis of hepatocellular carcinoma. J Int Med Res 46: 348-356, 2018.

15. Sui J, Yang X, Qi W, Guo K, Gao Z, Wang L and Sun D: Long non-coding RNA linc-USP16 functions as a tumour suppressor in hepatocellular carcinoma by regulating PTEN expression. Cell Physiol Biochem 44: 1188-1198, 2017. 
16. Hao QQ, Chen GY, Zhang JH, Sheng JH and Gao Y: Diagnostic value of long noncoding RNAs for hepatocellular carcinoma: A PRISMA-compliant meta-analysis. Medicine (Baltimore) 96: e7496, 2017.

17. Zhu J, Liu S, Ye F, Shen Y, Tie Y, Zhu J, Jin Y, Zheng X, Wu Y and $\mathrm{Fu} \mathrm{H}$ : The long noncoding RNA expression profile of hepatocellular carcinoma identified by microarray analysis. PloS One 9: e101707, 2014.

18. Tang J, Jiang R, Deng L, Zhang X, Wang K and Sun B: Circulation long non-coding RNAs act as biomarkers for predicting tumorigenesis and metastasis in hepatocellular carcinoma. Oncotarget 6: 4505-4515, 2015.

19. Cui H,Zhang Y, Zhang Q, Chen W, Zhao $\mathrm{H}$ and Liang J: A comprehensive genome-wide analysis of long noncoding RNA expression profile in hepatocellular carcinoma. Cancer Med 6: 2932-2941, 2017.

20. You J, Fang N, Gu J, Zhang Y, Li X, Zu L and Zhou Q: Noncoding RNA small nucleolar RNA host gene 1 promote cell proliferation in nonsmall cell lung cancer. Indian J Cancer 3 (Suppl 51): e99-e102, 2014.

21. Yu F, Bracken CP, Pillman KA, Lawrence DM, Goodall GJ, Callen DF and Neilsen PM: p53 Represses the oncogenic sno-MiR-28 derived from a snoRNA. PloS One 10: e0129190, 2015

22. Zhang M, Wang W, Li T, Yu X, Zhu Y, Ding F, Li D and Yang T: Long noncoding RNA SNHG1 predicts a poor prognosis and promotes hepatocellular carcinoma tumorigenesis. Biomed Pharmacother 80: 73-79, 2016.

23. Liu M, Xing LQ and Liu YJ: A three-long noncoding RNA signature as a diagnostic biomarker for differentiating between triple-negative and non-triple-negative breast cancers. Medicine (Baltimore) 96: e6222, 2017.

24. Page RD: TreeView: An application to display phylogenetic trees on personal computers. Comput Appl Biosci 12: 357-358, 1996.

25. Livak KJ and Schmittgen TD: Analysis of relative gene expression data using real-time quantitative PCR and the 2(-Delta Delta C(T)) method. Methods 25: 402-408, 2001.

26. Chaiteerakij R, Addissie BD and Roberts LR: Update on biomarkers of hepatocellular carcinoma. Clin Gastroenterol Hepatol 13: 237-245, 2015.

27. Benson AB, III, Abrams TA, Ben-Josef E, Bloomston PM, Botha JF, Clary BM, Covey A, Curley SA, D'Angelica MI, Davila R, et al: NCCN clinical practice guidelines in oncology: Hepatobiliary cancers. J Natl Compr Canc Netw 7: 350-391, 2009
28. Kong FY, Xu BY, Du Y, Xu JJ and Chen HY: A branched electrode based electrochemical platform: Towards new label-free and reagentless simultaneous detection of two biomarkers. Chem Commun (Camb) 49: 1052-1054, 2013.

29. Ma X, Wang X, Yang C, Wang Z, Han B, Wu L and Zhuang L: DANCR acts as a diagnostic biomarker and promotes tumor growth and metastasis in hepatocellular carcinoma. Anticancer Res 36: 6389-6398, 2016

30. Jing W, Gao S, Zhu M, Luo P, Jing X, Chai $\mathrm{H}$ and Tu J: Potential diagnostic value of lncRNA SPRY4-IT1 in hepatocellular carcinoma. Oncol Rep 36: 1085-1092, 2016.

31. Yuan W, Sun Y, Liu L, Zhou B, Wang S and Gu D: Circulating LncRNAs serve as diagnostic markers for hepatocellular carcinoma. Cell Physiol Biochem 44: 125-132, 2017.

32. Zhang H, Zhou D, Ying M, Chen M, Chen P, Chen Z and Zhang F: Expression of long non-coding RNA (lncRNA) small nucleolar RNA host gene 1 (SNHG1) exacerbates hepatocellular carcinoma through suppressing miR-195. Med Sci Monit 22: 4820-4829, 2016.

33. Yang SL, Liu LP, Yang S, Liu L, Ren JW, Fang X, Chen GG and Lai PB: Preoperative serum $\alpha$-fetoprotein and prognosis after hepatectomy for hepatocellular carcinoma. Br J Surg 103: 716-724, 2016.

34. Sahu D, Hsu CL, Lin CC, Yang TW, Hsu WM, Ho SY, Juan HF and Huang HC: Co-expression analysis identifies long noncoding RNA SNHG1 as a novel predictor for event-free survival in neuroblastoma. Oncotarget 7: 58022-58037, 2016.

35. Wang J, Cao L, Wu J and Wang Q: Long non-coding RNA SNHG1 regulates NOB1 expression by sponging miR-326 and promotes tumorigenesis in osteosarcoma. Int J Oncol 52: 77-88, 2018.

36. Zhang $Y$, Jin $X$, Wang Z, Zhang $X$, Liu $S$ and Liu G: Downregulation of SNHG1 suppresses cell proliferation and invasion by regulating notch signaling pathway in esophageal squamous cell cancer. Cancer Biomark 21: 89-96, 2017.

This work is licensed under a Creative Commons Attribution-NonCommercial-NoDerivatives 4.0 International (CC BY-NC-ND 4.0) License. 